\title{
Atención integral del paciente con labio y paladar fisurado
}

\section{Integral Treatment of pacients with cleft lip and palate}

\author{
Palacios Vivar Diego ${ }^{1 *}$, Alvear Córdova María Cristina ${ }^{2}$, Alvarado Cordero Alberto ${ }^{3}$, Reinoso Quezada Santiago ${ }^{1}$ \\ ${ }^{1}$ Especialista en Cirugía Oral y Maxilofacial \\ 2 Especialista en Odontopediatría \\ ${ }^{3}$ Especialista en Ortopedia y Ortodoncia \\ Docentes de la Carrera de Odontología, Especialistas de la Clínica de Labio y Paladar Fisurado, Universidad Católica de \\ Cuenca. Ecuador \\ *clínicalabio83@gmail.com
}

\begin{abstract}
Resumen
La atención del paciente con Labio y Fisura Palatina es compleja e implica involucrar múltiples proveedores de salud: cirujano, pediatra, otorrinolaringólogo, dermatólogo, ortopedista, ortodoncista, odontólogo pediátrico, psicólogo, fonoaudiólogo, entre otros; sumando un total recomendado de 18 especialistas. Con el tiempo se ha pasado de un modelo de atención individual intermitente en consultorio a un modelo multidisciplinario en una espacio físico especializado "clínica" en donde confluyen los especialistas para tratar al paciente de forma integral con un enfoque de equipo coordinado de atención continua.
\end{abstract}

Palabras clave: Labio fisurado, paladar fisurado, equipo multidisciplinario, cirugía, malformación.

\begin{abstract}
The care of the patient with Lip and Cleft Palate is complex and involves multiple health providers: surgeon, pediatrician, otolaryngologist, dermatologist, orthopedist, orthodontist, pediatric dentist, psychologist, speech therapist, among others; adding a recommended total of 18 specialists. Over time, it has gone from a model of intermittent individual care in the office to a multidisciplinary model in a specialized physical space "clinic" where specialists come together to treat the patient in a comprehensive way with a coordinated team approach and a continuous care.
\end{abstract}

Key words: Cleft lip, cleft palate, patient care team, surgery, malformation.

\section{INTRODUCCIÓN}

Las hendiduras orofaciales son defectos del nacimiento que constituyen grandes desafíos para la cirugía craneofacial y una serie de especialidades que brindan acompañamiento para proporcionar atención integral preventiva y terapéutica del paciente fisurado. Se estima que aproximadamente 3,5 millones de pacientes pediátricos en todo el mundo padecen esta afección. ${ }^{1}$ La carga de la enfermedad por parte de las familias y las barreras para la atención integral son evidentes, se necesitan diferentes servicios e intervenciones multidisciplinarias con tiempos críticos para lograr resultados que mejoren la calidad de vida. ${ }^{2}$ El tratamiento de pacientes con hendiduras orofaciales ha sido definido en la literatura incluso como una "empresa multidisciplinaria e interdisciplinaria", que ejerce sus funciones desde la etapa prenatal con el asesoramiento familiar y continúa desarrollando roles estratégicos que se extienden a lo largo de la vida del individuo.,
Reconocer que el manejo del paciente con Labio y Paladar Fisurado(LPF) es complejo, implica involucrar múltiples especialistas de la salud; cuya interacción es clave para estructurar un equipo de atención exitoso. ${ }^{1,5}$

\section{ESTADO DEL ARTE}

Los principios de cirugía (anestesia, colgajo, sutura) no han sufrido modificaciones en los últimos 50 años, lo que ha cambiado han sido los materiales, el manejo ortopédico, ortodóncico, implantes y técnicas quirúrgicas de avances óseos. ${ }^{6,7}$ Son pocas las técnicas quirúrgicas nuevas, lo que ha sucedido es que las técnicas clásicas se han perfeccionado y cada centro ha desarrollado modificaciones leves o moderadas de acuerdo a sus experiencias., ${ }^{4,5}$

En un inicio los esfuerzos de los especialistas de medicina y odontología se realizaban de manera individual para tratar al paciente con LPF. Actualmente el esfuerzo y manejo es en equipo, de forma que se logré garantizar 


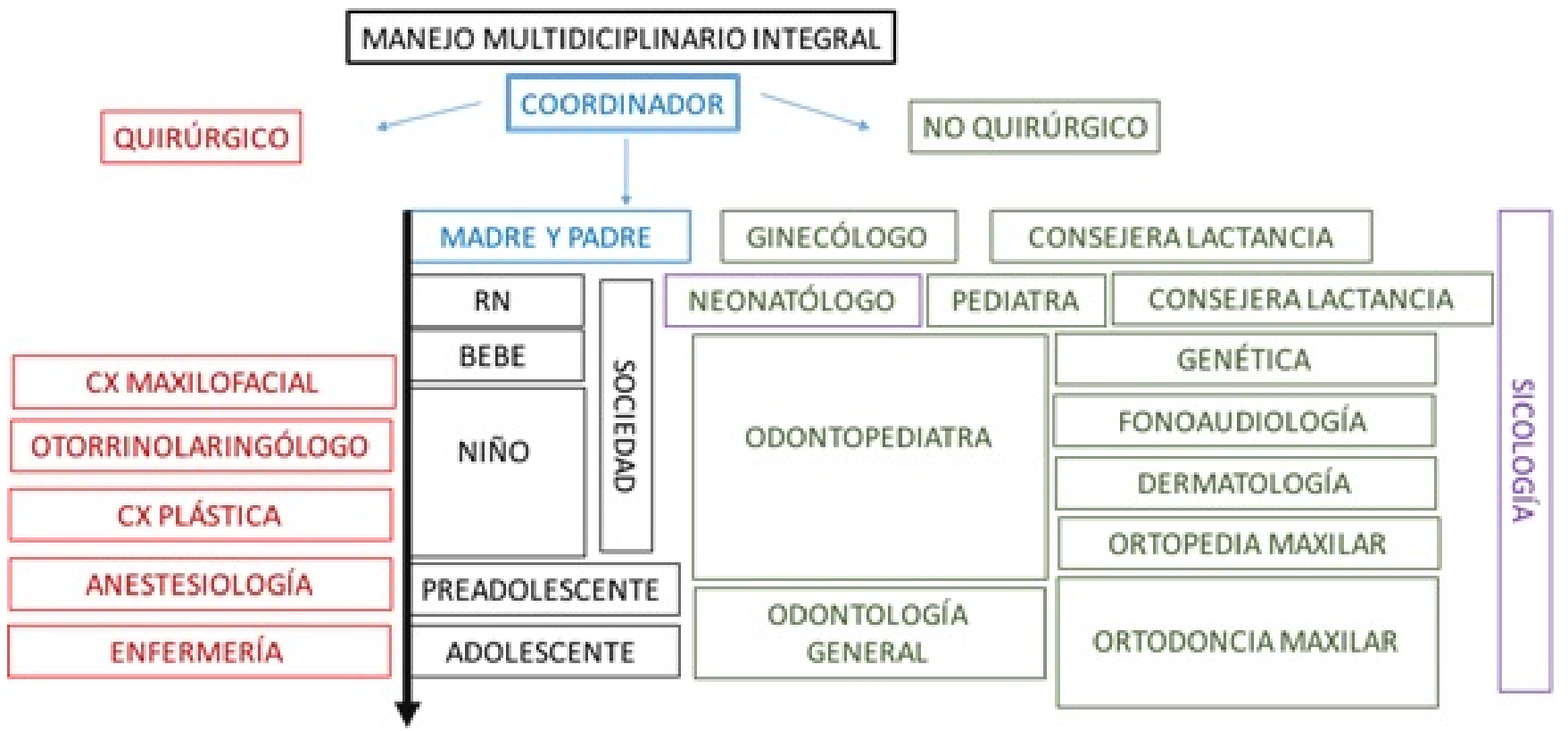

Fig. 1. Algoritmo Manejo multidisciplinario ${ }^{3-6,8}$ Manejo multidisciplinario integral compuesto de 18 especialistas para la atención del paciente con Labio y Paladar Fisurado en la Carrera de Odontología de la Universidad Católica de Cuenca. Fuente: Adaptación propia

una atención coordinada y consistente, con una secuencia adecuada de evaluaciones y tratamientos, considerando como una variable importante el crecimiento y desarrollo de cada paciente. ${ }^{6}$

En el pasado los pacientes tenían que deambular de consultorio a consultorio en diferentes lugares y en ocasiones distintos horarios dependiendo de la disponibilidad del profesional. En la actualidad las personas involucradas tienen acceso a clínicas especializadas en un horario con todos los profesionales trabajando en equipo. ${ }^{6}$

La nueva infraestructura de la Clínica de Labio y Paladar Fisurado de la Universidad Católica de Cuenca, ha logrado cambiar el esquema de tratamiento de campañas itinerantes por el modelo de atención permanente, canalizando los recursos materiales y servicios profesionales de la salud en un solo lugar. Lo que permite brindar atención especializada para los pacientes que requieran este tipo de tratamiento; con el compromiso de un esquema de tratamiento multidisciplinario integral, profesional y ético (Figura 1).

Los objetivos del tratamiento en niños con LPF engloban los siguientes aspectos 6

- Reparar defectos del nacimiento: labio, paladar, proceso alveolar, nariz.

- Alcanzar el equilibrio de todas las estructuras orofaciales que participan en la comunicación normal y desarrollo del habla.

- Establecer la estabilidad oclusal necesaria, así como los hábitos correctos de salud oral.

- Optimizar el aspecto psicosocial, así como el desarrollo social y familiar del niño.

- Proporcionar opciones económicas rentables para los tratamientos, los cuales son apoyados y auspiciados por organizaciones sociales y educativas como : Universidad Católica de Cuenca, Club Rotario Cuenca, Hospital Municipal de la Mujer y el Niño.

Los parámetros de atención a seguir contemplan las siguientes actividades 6

- Intervención temprana, prenatal o del recién nacido.

- Un primer diagnóstico interdisciplinario con todo el equipo de profesionales valorando la necesidad y secuencia de los siguientes tratamientos: quirúrgico, fonoaudiológico, odontopediátrico, ortodóntico, ortopédico, sicológico y cognitivo, entre otros.

- Actualizaciones y capacitación continua de los profesionales, en el manejo de pacientes con LPF.

- Tratamiento continuo y seguimiento longitudinal durante la niñez y la adolescencia.

- Selección adecuada de los tiempos quirúrgicos, por su significacia en el crecimiento facial, oclusión y lenguaje.

- Coordinación y reuniones periódicas del equipo para determinar todas las decisiones del tratamiento de manera integral e individualizada. 
- Disminuir la posibilidad de sobretratamientos, mejorando la rentabilidad de los costos para los padres.

Con el propósito de verificar la calidad de atención del equipo multidisciplinario, se ha establecido una serie de estándares a ser cumplidos, mismos que buscan el desarrollo profesional y colectivo mediante la investigación y actualización constante. Toda la información proporcionada a los pacientes, familiares o cuidadores en relación a los servicios y limitaciones que el equipo provee debe ser honesta $\mathrm{y}$ certera.

\section{DISCUSIÓN}

Actualmente los equipos multidisciplinarios constituye el estándar de oro para la atención de pacientes con LPF, es por esto que la carencia de este podría resultar en una serie de errores diagnósticos, tratamientos innecesarios y programación de tiempos sin cronicidad. $^{9}$ La Carrera de Odontología de la Universidad Católica de Cuenca en su afán de garantizar una atención coordinada, consistente y secuencial, ha agrupado profesionales experimentados y calificados en todas las áreas requeridas, quienes trabajan aliados dentro del marco de necesidades del paciente fisurado y bajo estándares internacionales de atención. ${ }^{8,9}$

Es importante realizar un registro escrito y detallado de las responsabilidades atribuidas a cada uno de los miembros, incluyendo las edades de atención e intervención quirúrgica. Una vez consolidado el equipo, el desarrollo de un protocolo o guía práctica de atención clínica resulta imprescindible para la toma de decisiones, así será más preciso conocer y aplicar la mejor opción en cada etapa de tratamiento.,

Un equipo multidisciplinario universitario debe integrar estudiantes y profesionales, en este caso los estudiantes cumplirán sus actividades de acuerdo a las destrezas adquiridas y los profesionales complementarán los tratamientos agregando su conocimiento y experiencia clínica. La obligación de la clínica de LPF no debería centrarse en la atención, sino en la educación de la comunidad sobre las características de estos pacientes y sus necesidades. ${ }^{6}$ El hecho de que a un paciente fisurado, se lo califique como " un caso complejo" no significa que todas las actividades que necesita serán de una complejidad alta; existirán actividades de complejidad menor que pueden ser realizadas por el estudiante del pregrado y otras por parte del posgradista, pero siempre supervisadas por el especialista. ${ }^{9,10}$

Respecto a los costos de un tratamiento de estos pacientes, se deberá considerar la situación socio económica de cada núcleo familiar, de esta manera se puede tener una aportación significativa para cubrir ciertos gastos materiales, ya que los honorarios son parte de una labor social; Debemos considerar que el mayor porcentaje de estos casos se presenta en personas de estratos bajos, por lo tanto, esto implicaría la intervención de fundaciones y del Estado para solventar gastos en general, tales como: infraestructura, materiales, medios diagnósticos, terapias, etc. ${ }^{8,10}$

Es necesario que la atención del paciente LPF se lleve a cabo en un solo lugar, y que este ejercicio este coordinado por una persona (logística) que permita una comunicación vertical y horizontal con los especialista entre si y la familia del paciente. $^{6}$ Tener un lugar de atención único, muestra notables beneficios como: evitar desplazamientos innecesarios, pérdidas de tiempo, inasistencias escolares, permisos de trabajo del familiar, etc. Para alcanzar esta meta es también importante la atención de todos los profesionales en un mismo horario y día. ${ }^{4}$

\section{CONCLUSIONES}

Estándares actuales de atención se pueden alcanzar y solventar, siempre y cuando los especialistas apropiados trabajen conjuntamente en interacción para estructurar el interrogatorio, diagnóstico y plan de tratamiento del paciente con LPF. La atención en equipo proporcionará resultados clínicos eficientes y resultará la opción económica más rentable para el paciente. La responsabilidad de la Carrera de Odontología a través de su equipo multidisciplinario en la Clínica de Labio y Paladar Fisurado es proveer recursos, materiales odontológicos, así como la infraestructura necesaria y moderna para los pacientes, padres y estudiantes, estos últimos involucrados cada vez más en procesos preventivos, restauradores, interceptivos e investigativos. Las intervenciones diseñadas a través del equipo multidisciplinario se enfocarán en obtener los mejores resultados, en el menor tiempo posible y en todas las etapas del tratamiento para este fin, se dispone de una infraestructura moderna que permite la realización de múltiples consultas en un solo día. El rol especifico de cada uno de los profesionales es documentado en la historia clínica del paciente, de ahí la importancia que la capacitación de todos los miembros del equipo sea evidente en términos de idoneidad y experiencia clínica, para permitir un desarrollo organizado.

Después de una evaluación diagnóstica es lógico pensar que los padres podrían experimentar una amplia gama de emociones y dudas, el comunicarse constantemente con los pacientes y sus representantes, a través de un vínculo de confianza mutua nos permitirá ayudar y asesorar en la toma de decisiones oportunas para el niño fisurado. La información que se brinde deberá contemplar la recomendación de cada uno de los tratamientos, las alternativas y los factores de riesgo asociados. En algunos casos la comunicación se puede establecer de manera directa con pacientes suficientemente maduros o adolescentes, quienes ya son alentados a participar en la toma de decisiones tanto por el clínico, como por sus padres. 
Cada paciente evaluado por el equipo recibe una planificación integral, con periodicidad continua $\mathrm{y}$ seguimiento longitudinal . La discusión de cada uno de los casos en un ambiente educativo y clínico a través de reuniones continuas de los profesionales garantiza la coordinación en la atención, logrando en los niños buenos perfiles faciales, simetría nasal, sonrisas atractivas, oclusiones armónicas y estructuras orales saludables; siendo estos objetivos conjuntos para todos los que participamos en la atención de un niño fisurado, nuestro compromiso es evidentemente clínico a nivel personal, pero su impacto involucra una promesa social y cultural para nuestros pacientes.

\section{Agradecimientos:}

A la contribución de nuestros alumnos de vinculación a la comunidad: Marcia Paola Albarracin Pintado, Diana Elizabeth Buele Yunga, Karen Priscila Sanchez Pinos, Alejandra Gabriela Ruiz Hurtado, Steven Alberto Saldarriaga Armijos, Maria Sol Medina Abad.
CONFLICTO DE INTERESES: Los autores no manifiesta ningún conflicto de interés.

\section{Referencias Bibliográficas}

1 Kling RR, Taub PJ, Ye X, Jabs EW. Oral clefting in China over the last decade: 205.679 patients. Plast Reconstr Surg Glob Open. 2014;2(10):e236.

2 Basseri B, et al.Current national incidence, trends, and health care resource utilization of cleft lip-cleft palate. Plast Reconstr Surg. 2011;127(3):1255-62.

3 Navarrete-Hernández E., Canún Serrano S, ValdésHernández J, Reyes-Pablo A.Prevalencia de labio hendido con o sin paladar hendido en recién nacidos vivos. México, 2008-201. Rev Mex pediatr. 2017; 84(3): p. 101-10.

4 Téllez C, Escobar LM, González MC, Moreno PS, Mora II, Bautista GR, Rojas AP. Detección clínica de anomalías craneofaciales mayores y menores en población escolar colombiana. Univ Odontol. 2014 Ene-Jun; 33(70): 65-77.

5 Teissier N, Bennaceur S, Van Den A.Tratamiento primario del labio leporino y paladar hendido. EMC. 2016 Abril; 17 (1): p. 1-14.

6 American Cleft and Palate- Craneofacial Organization Standards for Approval of Cleft Palate and Craniofacial Teams. 2019.

7 Akram A, McKnight MM, Bellardie H, Beale V, Evans RD. Craniofacial malformations and the orthodontist. British dental Journal. 2015 Febrero; 218(3).

8 Rubio Álvarez L. Defectos del habla relacionados con maloclusiones dentales, en niños con labio y/o paladar hendido no sindrómico en dentición mixta, de la fundación "Niños que ríen", República Dominicana. Universidad Nacional de Colombia. 2017.

9 Matovelle C, Matovelle P, Martínez F, Córdova Neira F.Estudio Descriptivo: Frecuencia de Malformaciones Congénitas en Pacientes Pediatricos del Hospital "José Carrasco Arteaga". Rev Med HJCA. 2015; 7(3): p. 249-53.

10 García Vidal DC.Prevalencia de labio y/o paladar hendido en pacientes de consulta externa del hospital Vicente Corral Moscoso en el periodo octubre 2011 - octubre 2016. Trabajo de Titulación previo a la obtención del Título de Médica. 2017.

Recibido: 10 de Enero del 2020

Aceptado: 22 de Abril del 2020 